\title{
e-Phaïstos
}

e-Phaïstos

Revue d'histoire des techniques / Journal of the history

of technology

IV-2 | 2015

Patrimoines de l'eau

\section{Eau et énergie : l'aqueduc de Ferrari Galliera dans le réseau des aqueducs de la ville de Gênes}

Water and power: the aqueduct of Ferrari Galliera in the network of aqueducts of Genoa

\section{Alberto Manzini}

\section{(2) OpenEdition}

Journals

Édition électronique

URL : http://journals.openedition.org/ephaistos/736

DOI : 10.4000/ephaistos.736

ISSN : 2552-0741

Éditeur

IHMC - Institut d'histoire moderne et contemporaine (UMR 8066)

Édition imprimée

Date de publication : 1 octobre 2015

Pagination : 22-35

ISSN : 2262-7340

Référence électronique

Alberto Manzini, «Eau et énergie : I'aqueduc de Ferrari Galliera dans le réseau des aqueducs de la ville de Gênes », e-Phaïstos [En ligne], IV-2 | 2015, mis en ligne le 28 novembre 2016, consulté le 01 mai 2019. URL : http://journals.openedition.org/ephaistos/736 ; DOI : 10.4000/ephaistos.736 


\section{Eau et énergie : I'aqueduc De Ferrari Galliera dans le réseau des aqueducs de la ville de Gênes}

\begin{abstract}
Introduction
Le territoire de la région Ligurie - nord-ouest de l'Italie - présente toutes les caractéristiques de l'environnement apenninique: montagnes aux pentes raides, torrents de portée irrégulière, étés secs et hivers très froids sur les hauteurs. La ville portuaire de Gênes, pointe septentrionale du golf homonyme, au pied de la chaîne de montagnes est confrontée depuis l'époque romaine au problème d'approvisionnement en eau. Pendant longtemps, cette question a été résolue par un seul aqueduc, communément appelé "aqueduc historique», plusieurs fois agrandi et amélioré au moyen âge et à l'époque moderne mais dépendant de sources sujettes à variation de niveau tout le long de l'année. Les transformations qui s'enchaînent avec l'industrialisation au XIX ${ }^{\mathrm{e}}$ siècle, la forte augmentation de la population urbaine et l'essor industriel des territoires côtiers et apenniniques imposent la recherche de solutions qui puissent garantir une quantité d'eau stable et suffisante pour la population - en lien avec la nouvelle attention pour l'hygiène - et répondre également aux nécessités de l'industrie naissante.

Une occasion inattendue se présente lorsqu'en creusant le tunnel apenninique du chemin de fer Gênes-Turin (1845-1853), les eaux du torrent
\end{abstract}

\author{
Alberto Manzini \\ Doctorant auprès de la Scuola \\ Superiore in Studi Storici, geografici e \\ antropologici delle Università di \\ Verona, Padova e Venezia, \\ Master Erasmus Mundus TPTI, \\ promotion 4 « Agora TPTI ».
}

Scriva, qui courait au nord de la chaîne de montagne, deviennent accessibles. Les eaux distribuées par l'Aqueduc Nicolay de 1853 représentent la première exploitation du bassin versant au nord de l'Apennin en démontrant la faisabilité de cette solution. Dans les années suivantes, grâce aux possibilités offertes par le développement de l'ingénierie (notamment dans la construction des barrages), de nombreuses études sont conduites pour rechercher un endroit dans la région montagneuse qui contourne la ville, adapté à la réalisation d'un lac artificiel. Enfin, sera retenu le projet présenté en 1871 par les ingénieurs Niccolò Burno et Stefano Grilli, pour la rivière du Gorzente, qui comprend tant la distribution de l'eau que la volonté d'exploiter la chute pour la production d'énergie dédiée à l'industrie en Val Polcevera (Nord-Ouest de Gênes).

Cette contribution, en tirant profit de la disponibilité de la documentation archivistique et du récent intérêt historiographique pour ce thème, veut faire ressortir des aspects patrimoniaux patrimoine de l'eau, patrimoine industriel et patrimoine technique - de l'aqueduc De Ferrari Galliera, en analysant son histoire et concentrant l'attention sur les structures aval du bassin. Ainsi, nous pouvons comprendre le double rôle qu'il a joué - fournir eau et énergie - et faire émerger des 
éléments de cet important patrimoine (encore en partie caché) qui deviennent les clés de lecture de l'évolution rapide du territoire apenninique de la ville de Gênes, centre d'avant-garde dans l'industrialisation de l'Italie entre les XIXe et XXe siècles.

Nos points de départ seront l'aqueduc historique et celui de Nicolay (1853) avec lesquels nous introduisons la spécificité du cas de Gênes et les exigences auxquelles la ville se trouve confrontée dans la seconde partie du XIX ${ }^{\mathrm{e}}$ siècle. Ensuite, nous traitons les premières phases de l'aqueduc De Ferrari Galliera et la réalisation de la ligne télédynamique pour l'industrie textile à Isoverde. Nous concluons enfin par les travaux complexes des trois centrales hydroélectriques liées au réseau de distribution en courant continu et la construction de la nouvelle centrale hydroélectrique.

\section{De l'aqueduc historique à la ville industrielle}

L'aqueduc historique, ainsi appelé car en usage depuis le moyen âge, fournit Gênes en utilisant des sources localisées dans la partie haute de la vallée Bisagno, qui se développe à l'Est de la ville. L'aqueduc, encore aujourd'hui en bonne partie visible et accessible comme parcours pour les randonneurs, suit le côté occidental de la vallée sur une distance d'environ $28 \mathrm{~km}$. L'itinéraire initial de l'aqueduc, développé sous l'Empire romain, d'une longueur d'environ $11 \mathrm{~km}$, est substitué par celui visible aujourd'hui et construit autour du XI ${ }^{\mathrm{e}}$ siècle à un niveau plus élevé que le précédent, permettant d'atteindre les parties les plus hautes de la ville. Le parcours de l'aqueduc a subi de nombreuses modifications au fil du temps en raison de sa localisation et de la nécessité de satisfaire la demande croissante en eau : si la vallée du Bisagno pouvait assurer une plus grande disponibilité d'eau - par rapport à la vallée du Polcevera - de nombreux ouvrages d'art (ingénierie) furent construits pour pallier aux problèmes de stabilité du terrain. Durant le XVIIe siècle, avec l'amélioration du réseau et des prises d'eau, les ouvrages réalisés par l'architecte Giovanni Aicardo (1622-1639) comprennent la construction de quarante pont-canaux, dont celui (toujours existant) du Rio Torbido d'une longueur de $130 \mathrm{~m}$ et d'une hauteur de $35,40 \mathrm{~m}$ construit en 1623. L'importance acquise par l'aqueduc apparait dans le travail commandé par les magistrats de la ville au cartographe Matteo Vinzoni (1690-1773), à l'occasion de la restauration de l'aqueduc en 1728 . Vinzoni, également auteur des plans des domaines de la République, élabore un atlas de dix grandes planches, destinées à un système de contrôle technique, illustrant les dix sections de l'aqueduc entre la prise et les murs de la ville destinées, cela afin d'éviter toute forme d'abus ${ }^{1}$.

La fin du XVIII ${ }^{\mathrm{e}}$ siècle voit s'achever l'expérience pluriséculaire de la république aristocratique de Gênes ; et avec la république ligurienne et ensuite la création du département de Gênes lors de son annexion à l'Empire français, la perte du pouvoir central de la ville au profit d'autres centres régionaux, liée au changement dans la gestion politique de la ville et du territoire. Après 1815, avec l'annexion au Royaume de Sardaigne, la classe dirigeante génoise et l'économie de la ville se trouvent encore plus marginalisées, un mécontentement croissant aboutit à l'insurrection de 1849. Suite à cet évènement, le Royaume de Sardaigne essaie de favoriser l'insertion de l'aristocratie et de la bourgeoise génoises dans l'économie sarde.

$\mathrm{Au}$ début du siècle, la région, à l'économie essentiellement agricole - faible et peu rentable sans compter les ports commerciaux de Gênes et Savone - devint dans la seconde partie du siècle, grâce à l'intervention de l'Etat et à la disponibilité de capitaux génois, un lieu de fort investissement ; les entrepreneurs ayant accès à un bassin de main d'œuvre à bas coût pour les premières manufactures, puis les usines qui apparaissaient autour de la ville et dans les vallées apenniniques ${ }^{2}$. Les sociétés basées à Gênes relèvent d'intérêts 
locaux liés au nouvel état et à l'échelle internationale: de la Monteponi créée pour l'exploitation des mines d'argent et de plomb en Sardaigne aux entreprises qui se développent dans l'espace urbain (et dans les villes limitrophes de Sampierdarena et Sestri Ponente) où se multiplient les usines mécaniques modernes comme celles des entrepreneurs, italiens comme Giovanni Ansaldo et Luigi Orlando, ou étrangers comme Thomas Robertson, Alessandro Westerman, Joseph Marie Balladyer, John Wilson et Alexander Maclaren, qui redessinent la physionomie des alentours de la ville 3 .

Ce rapide développement va de pair, dans les années du milieu du siècle, avec la construction du chemin de fer Gênes-Turin qui, inauguré en 1853, fait du port de la ville le premier en liaison directe avec la plaine padane et les villes industrielles du Nord de la péninsule italienne (le transport de marchandises dépasse rapidement les 548640 tonnes par de l'année 1845 pour atteindre 750 ooo en 1850 et 905000 en 1861 après l'ouverture de la ligne ferroviaire).

La nouvelle ville industrielle, au barycentre déplacé du vieux centre historique vers l'Ouest et en direction des vallées apenniniques de Polcevera, de Verde, de Scrivia et de Stura, voit augmenter ses exigences d'eau jusqu'alors soutenues par le seul aqueduc historique. $\mathrm{Au}$ milieu du siècle, l'opportunité de résoudre ce problème se présente lors du creusement du tunnel de Giovi (terminé en 1853), qui liait les deux versants de l'Apennin, avec l'émergence de nombreuses sources d'eau. Cet ouvrage d'art de 3250 mètres de long, extraordinaire pour l'époque, garde pour quelques années le primat mondial de la longueur de tunnel ferroviaire, suscitant l'intérêt du pays entier et des entrepreneurs génois intéressés par les nouvelles possibilités offertes de liaison avec le côté nord de l'Apennin. Les premiers projets, comme celui de l'architecte Novelli qui propose un aqueduc pour la ville alimenté avec les eaux du fleuve Scrivia4, se voient refuser la concession du gouvernement.
L'entrepreneur Paolo Antonio Nicolay réussit à convaincre le gouvernement : il demande et obtient la concession pour l'usage des eaux revenues dans le tunnel en accord avec la société ferroviaire qui établit que 1/10 soit attribuée aux chemins de fer en échange de l'utilisation de la galerie de Giovi 5.

Nicolay fonde en 1853 la compagnie du Nuovo Acquedotto, dont il est président; l'initiative, soutenue par Cavour et par les principaux personnages de la bourgeoisie génoise, se jouant des difficultés bureaucratiques, obtient l'autorisation pour la construction d'un aqueduc entièrement privé ${ }^{6}$ dont le débit est initialement établi à 350 litres par seconde7.

\section{La construction de l'aqueduc De Ferrari Galliera}

La progression de l'industrie en Ligurie s'accompagne de « la deuxième révolution industrielle » : innovation technologique (acier, électricité, nouvelle industrie chimique), intégration des marchés, expansion du PIB). Les choix économiques du parlement, en direction du protectionnisme (tarifs douaniers et commandes d'état), favorisent le monde entrepreneurial ligurien qui en profite dans le secteur sidérurgique. À la fin du siècle, sous plusieurs aspects, le triangle industriel Gênes-Turin-Milan ne se différencie pas des pays les plus avancés de l'Europe occidentale. La croissance du secteur secondaire est élevée tant dans le chef-lieu de Gênes que sur le territoire de la région entière ${ }^{8}$. La ville de la seconde moitié du

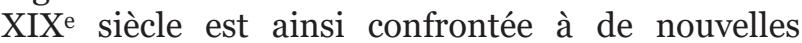
exigences, les entreprises grandissent, les vallées s'industrialisent, la population de la ville et des petits bourgs augmente. De nombreuses solutions sont proposées dans ces années pour la construction du troisième aqueduc; mais entre toutes, c'est celle des ingénieurs Niccolò et Salvatore Bruno et Stefano Grillo, présentée en 1871, qui semble le plus solide et finançable9. Le projet pour le nouvel aqueduc, qui tire profit de la 
solution déjà employée dans le cas du Nicolay (percer ou creuser l'Apennin et porter l'eau du versant nord au versant sud) est, selon les candidats, pertinent pour satisfaire les exigences de la ville ${ }^{10}$. La localité choisie pour la construction d'un grand bassin pour l'aqueduc se trouve aux limites les plus externes du versant nord de la vallée apennine génoise, dans l'actuelle vallée du lac Lungo et du Lac Lavezze, à l'origine du fleuve du Gorzente en haut du village d'Isoverde ${ }^{11}$ (mairie de Campomorone) ${ }^{12}$. La structure géologique du territoire, avec une extension d'environ 1700 hectares, garantit l'imperméabilité du fonds et son altitude - $680 \mathrm{~m}$ au-dessus du niveau de la mer permet de produire la force motrice nécessaire pour le fonctionnement de l'industrie locale.

Le projet proposé par les ingénieurs prévoit : un barrage de 35 mètres de hauteur (dans la vallée du Gorzente en localité de Lavezze) afin d'obtenir un réservoir de 2,4 millions de $\mathrm{m}^{3}$ d'eau, un bâtiment pour la gestion des dérivations de l'eau, un tunnel à travers l'Apennin pour amener l'eau sur le côté méridional de la chaîne et une canalisation (de 600 $\mathrm{mm}$ de diamètre et $20 \mathrm{~km}$ de longueur) jusqu'au centre-ville. La concession est obtenue le 23 Décembre 1873 après avoir résolu les problèmes de fourniture d'eau en aval du nouveau barrage ${ }^{13}$.
La double fonction du nouveau complexe distribution d'eau et d'énergie - émerge déjà dans les premiers documents disponibles quand, afin de recenser les nécessités énergétiques des industriels de la vallée Polcevera, les concessionnaires adressent le 28 novembre 1874 cette lettre au maire de Campomorone.

«[...] Les capitalistes anglais qui ont pris l'engagement de donner suite au projet du magnifique aqueduc du Gorzente demandent aux concessionnaires qu'ils leur indiquent dans quelles zones serait utilisée la force motrice obtenue avec l'aqueduc. [...] nombreuses ont été les adhésions [...]. Il est intéressant, cependant, que la force soit demandée le plus proche possible du point de production en évitant les coûts dérivants de canalisations ultérieures, les souscrits s'adressent à la gentillesse de votre seigneurie illustre afin que vous preniez contact avec les industriels de Campomorone pour indiquer aux concessionnaires le lieu et l'ampleur de la force nécessaire [...]. Il suffit que les industriels utilisassent un courrier adressé aux soussignés selon la forme qui a été utilisée par monsieur Sciaccaluga, les frères Figari et d'autres ${ }^{14}$.

Le courrier est complété par le texte qui pouvait être utilisé pour indiquer la quantité d'énergie nécessaire et le lieu d'implantation de l'usine afin que la Société De Ferrari Galliera puisse organiser au mieux les canalisations ${ }^{15}$.

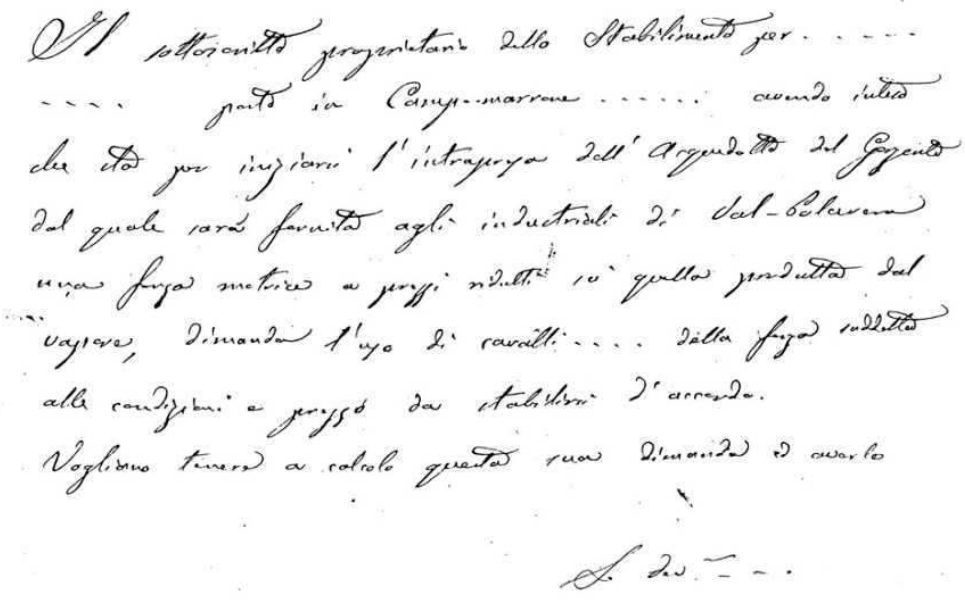

Figure 1 : Lettre d'exemple présentée en 1874 par les concessionnaires de l'aqueduc aux entrepreneurs du val Polcevera 
Les concessionnaires veulent atteindre deux objectifs, rassurer les investisseurs, dont on relève une référence assez vague dans le courrier, à propos de la vente de l'énergie produite; et organiser le réseau de distribution. Suite à plusieurs rénovations de la concession, ils impliquent les belges Graechen et Henschlinget et arrivent à réunir un groupe d'investisseurs ${ }^{16}$. Le 12 Février 1880 est fondée la Società Anonima Aqueduc De Ferrari Galliera ainsi nommée à la mémoire de la famille homonyme génoise réputée pour ses œuvres de philanthropie ${ }^{17}$. Dans le conseil d'administration se trouvent, entre autres, Niccolò Bruno, les héritiers de l'ingénieur Grillo, le marquis Lazare Negrotto Cambiaso et des représentants du monde entrepreneurial génois. Les travaux commencent la même année avec la réalisation du barrage de Lavezze et celui de Lavagnina pour terminer à la moitié de la décennie avec la mise en fonction de la prise d'eau et du tunnel de $2300 \mathrm{~m}$ qui traversent la montagne et portent l'eau sur le versant sud à une altitude de $622 \mathrm{~m}$ en haut du village d'Isoverde.

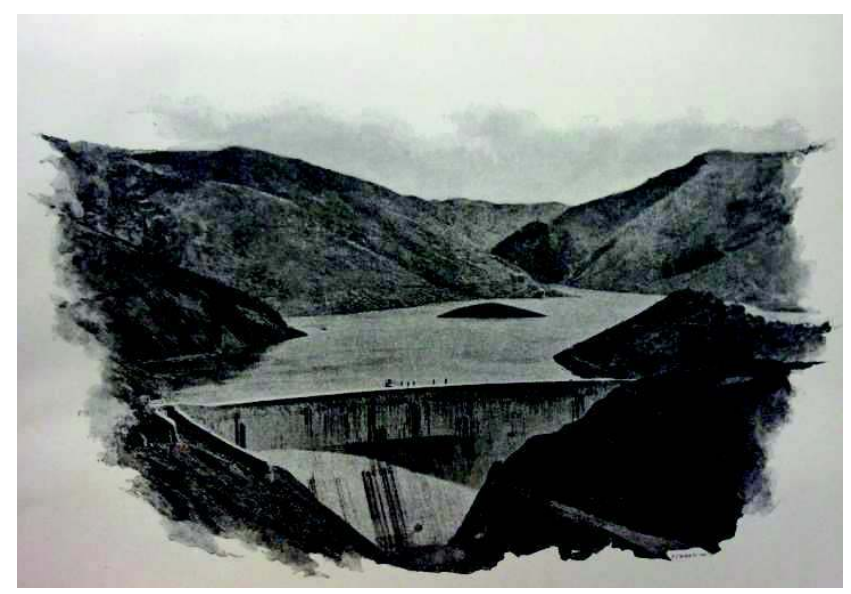

Figure 2 : Barrage de Lavezze, fin XIXe siècle (BRUNO Niccolò, L'acquedotto De Ferrari Galliera, Hoepli, Milano, 1893. Vol II. Annexe. A.)

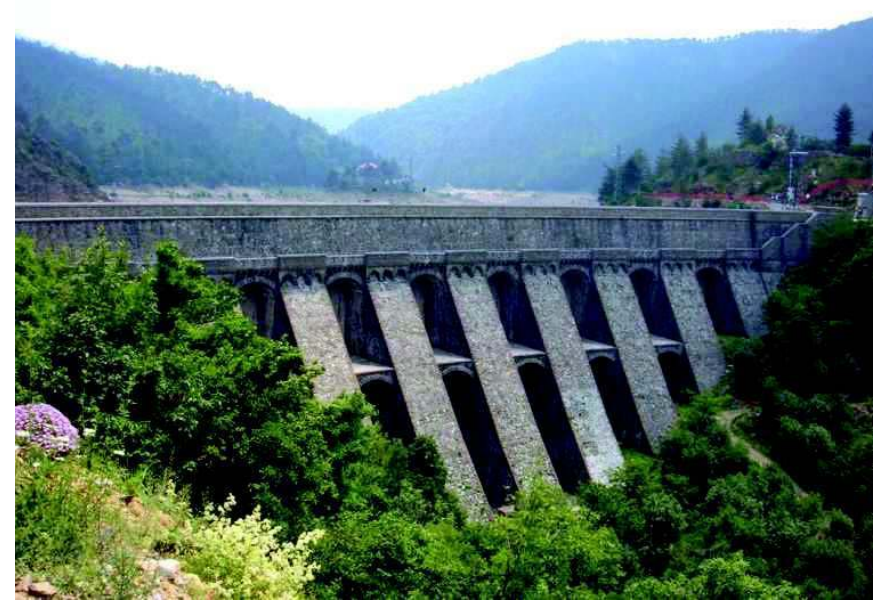

Figure 3 : Barrage de Lavezze, 2012 (photo Alberto Manzini, 2012)

\section{Première solution pour la distribution de l'énergie : la ligne télédynamique}

Les très bas taux d'émigration ainsi que la forte présence des personnels provenant d'autres provinces italiennes, qui dépasse le nombre de génois, selon les listes des travailleurs des chantiers de l'aqueduc, montrent, comme le relève Angelini, la vitalité industrielle de cette vallée ${ }^{18}$.

$\mathrm{Si}$, dès les premières phases du projet, les concessionnaires avaient pris en considération les exigences des entreprises en aval pour la distribution d'eau et d'énergie hydraulique, la première requête importante arrive en 1885 émanant de l'entrepreneur Costa qui avait l'intention d'implanter une filature à Isoverde, à proximité de la prise d'eau destinées à la canalisation directe à la ville. L'entreprise demande une puissance de 400 chevaux mais la localisation de l'usine, 50 mètres en aval de la prise d'eau, aurait obligé à détourner une partie des eaux au détriment du réseau principal direct vers Gênes. La société De Ferrari Galliera dispose alors la construction d'une 


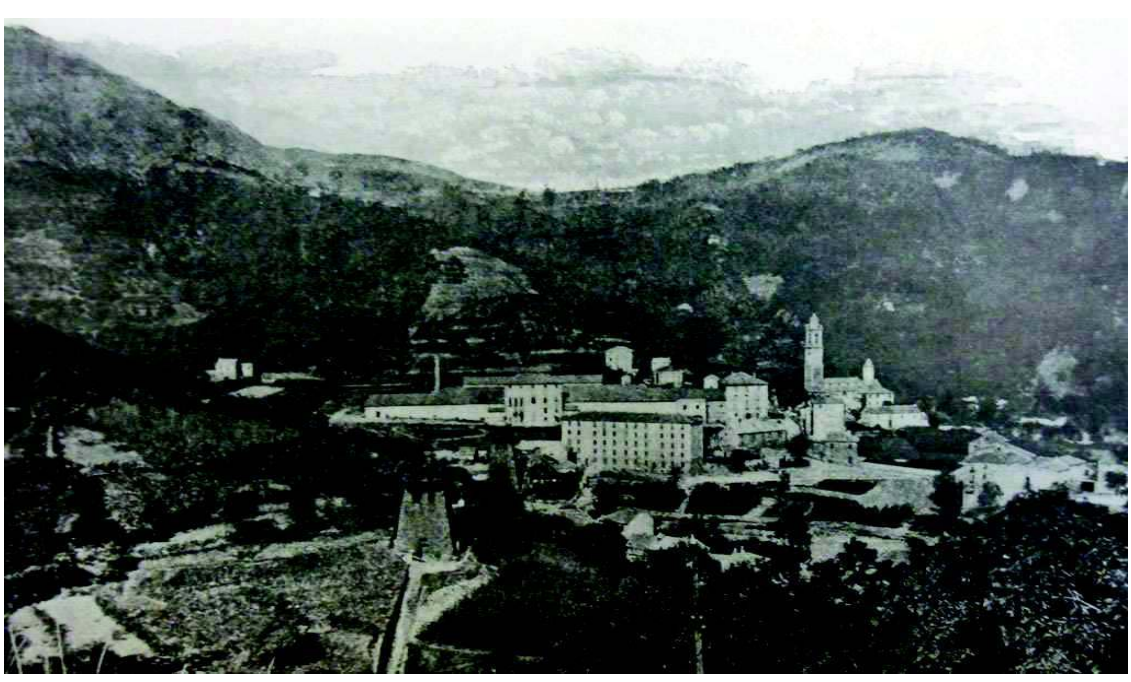

Figure 4: Le réseau télédynamique et l'usine Costa vus de la centrale hydromécanique.

centrale hydromécanique dans une position plus favorable pour la prise d'eau et la relie à l'entreprise par un système télédynamique de transport de l'énergie, long d'environ 400 mètres. Le complexe, terminé en 1886, se compose d'un bâtiment (14,70 x 12 mètres) accueillant les deux machines construites par l'entreprise J. Jacob Reiter - C. de Winterthur, le local technique pour la gestion de la pression de l'eau et un logement pour le chef d'atelier. Les deux piliers pour supporter les câbles, construits en pierres et enduits, sont localisés à 155,75 mètres de la centrale ; le premier est à $149,50 \mathrm{~m}$ du premier et le second à $96,75 \mathrm{~m}$ de l'usine Costa, pour un parcours total de 402 mètres. Chaque pilier est surmonté par le mécanisme de transmission du mouvement, obtenu grâce à deux grandes roues de 5 mètres de diamètre où passent les câbles en acier ${ }^{19}$.

\section{Les centrales \\ hydroélectriques - Galvani, Volta et Pacinotti - et l'essai du réseau à courant continue}

Étant donné que la production d'énergie de la première centrale est supérieure à celle demandée par la filature, la société De Ferrari Galliera destine son projet de fourniture d'énergie aux usines de la vallée Polcevera. L’ingénieur Alberto Preve propose, pour rejoindre les entreprises localisées tout le long de la vallée du Polcevera jusqu'au centre-ville, suite à la consultation de personnes «compétentes sur

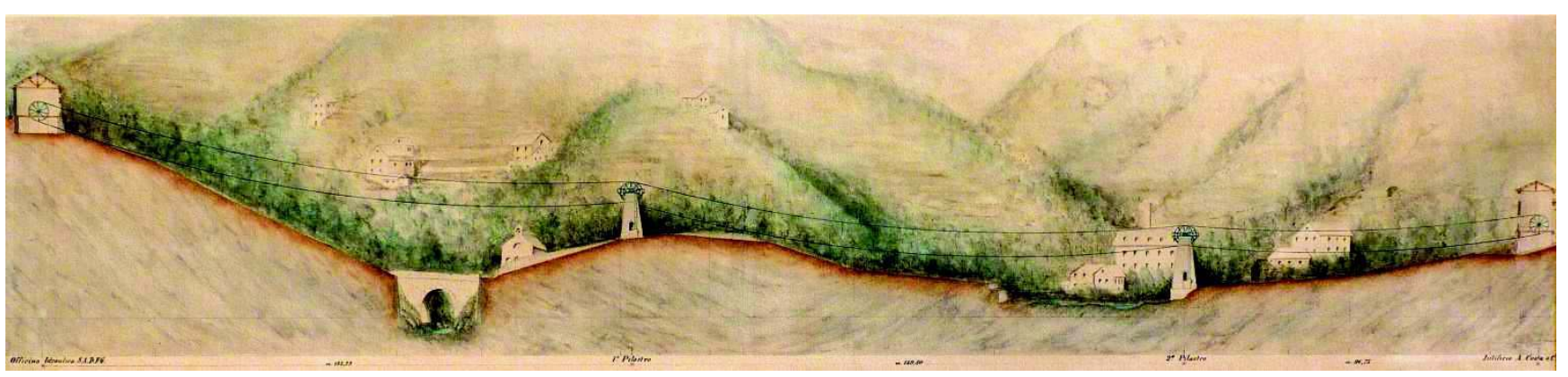

Figure 5: Finocchietti Ettore, Esquisse de la ligne télédynamique, 1884, Archive de la Fondazione AMGA 


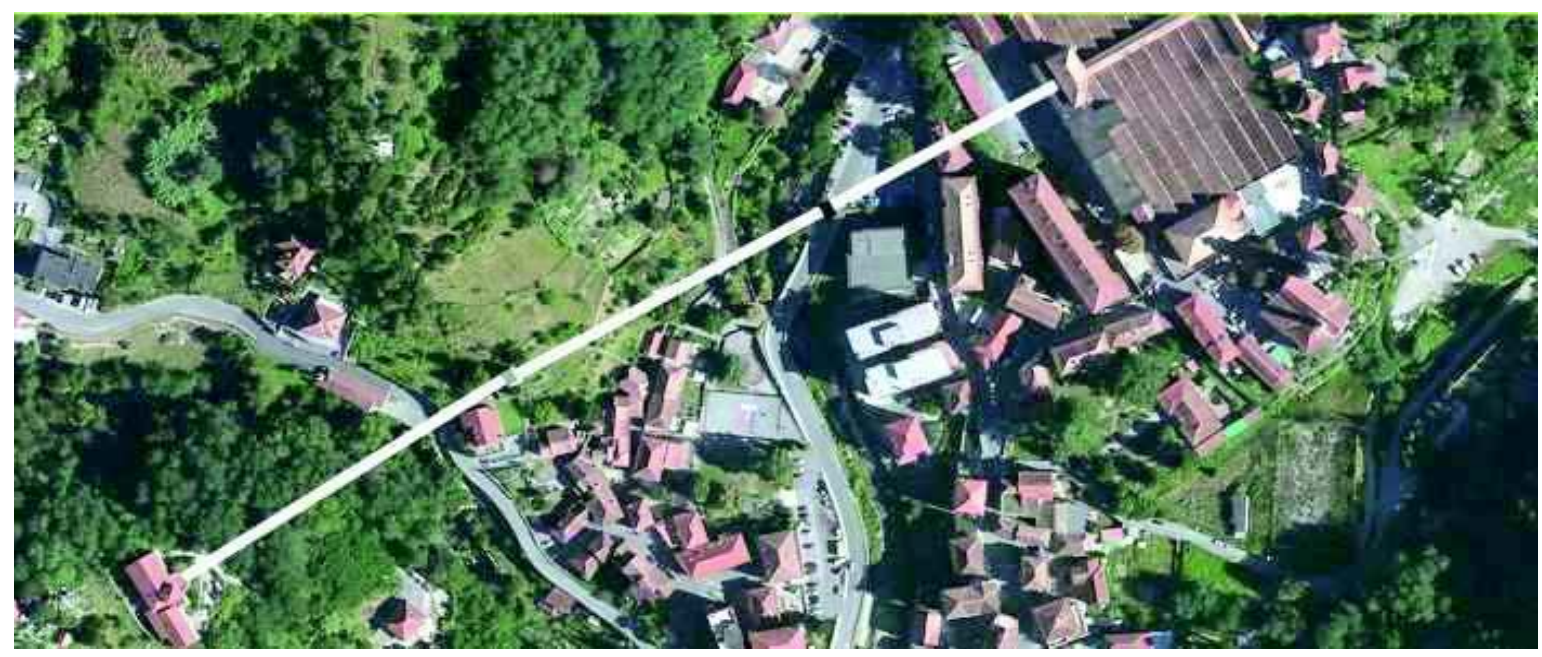

Figure 6 : Elaboration du parcours de la ligne télédynamique sur les photographies de Bing Maps.

l'électricité ", d'utiliser le système de transport à travers un courant continu, une méthode à l'époque déjà expérimentée pour l'illumination des villes américaines ${ }^{20}$. La réalisation de l'implantation présente de nombreuses difficultés techniques qui poussent la société génoise à contacter l'ingénieur Thury de la Société de l'Industrie Electrique de Genève. L’ingénieur genevois développe ainsi des solutions ingénieuses, permettant une distribution réalisable et rentable ${ }^{21}$. Le premier essai de 1889 donne un résultat positif et permet la réalisation de la grande centrale Galvani en 1890, à 253 mètre d'élévation, qui englobe la précédente centrale télédynamique.

La société réalise alors, afin d'exploiter toute la chute disponible, deux autres centrales (comme la première, dédiées aux grands hommes de sciences italiens): la Volta à 402 mètres avec 110 mètres de chute et la Pacinotti à 514 mètres avec 104 mètres de chute.

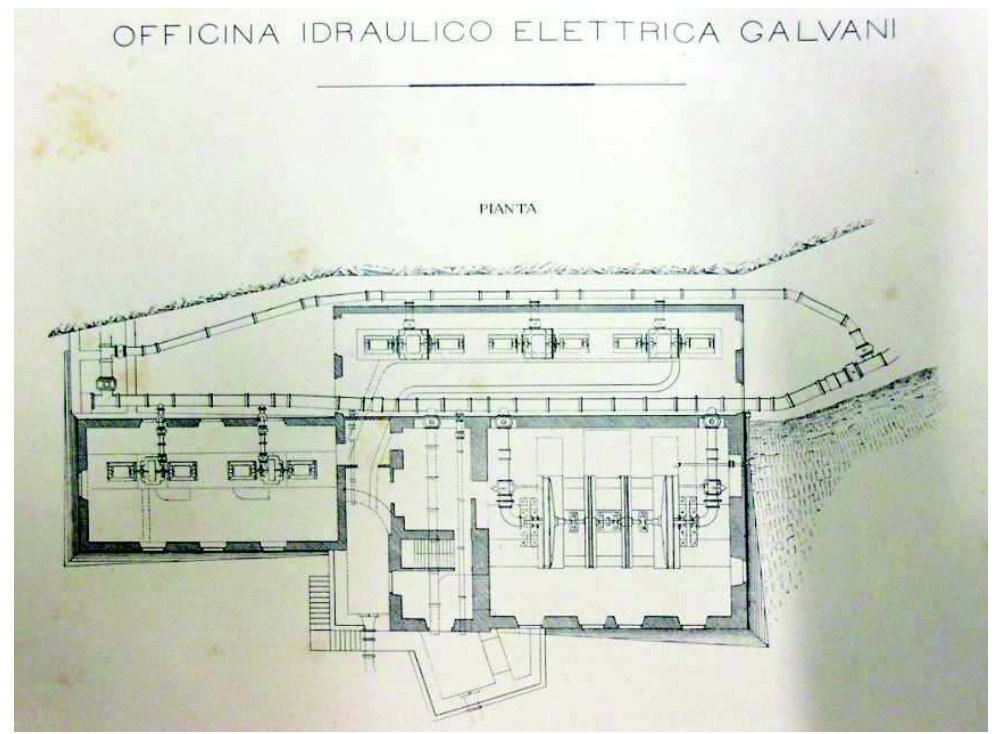

Figure 7 : Planimétrie de la centrale Galvani en 1893 (BRUNO Niccolò, L'acquedotto De Ferrari Galliera, Hoepli, Milano, 1893. Vol II. Annexe XXVI) 


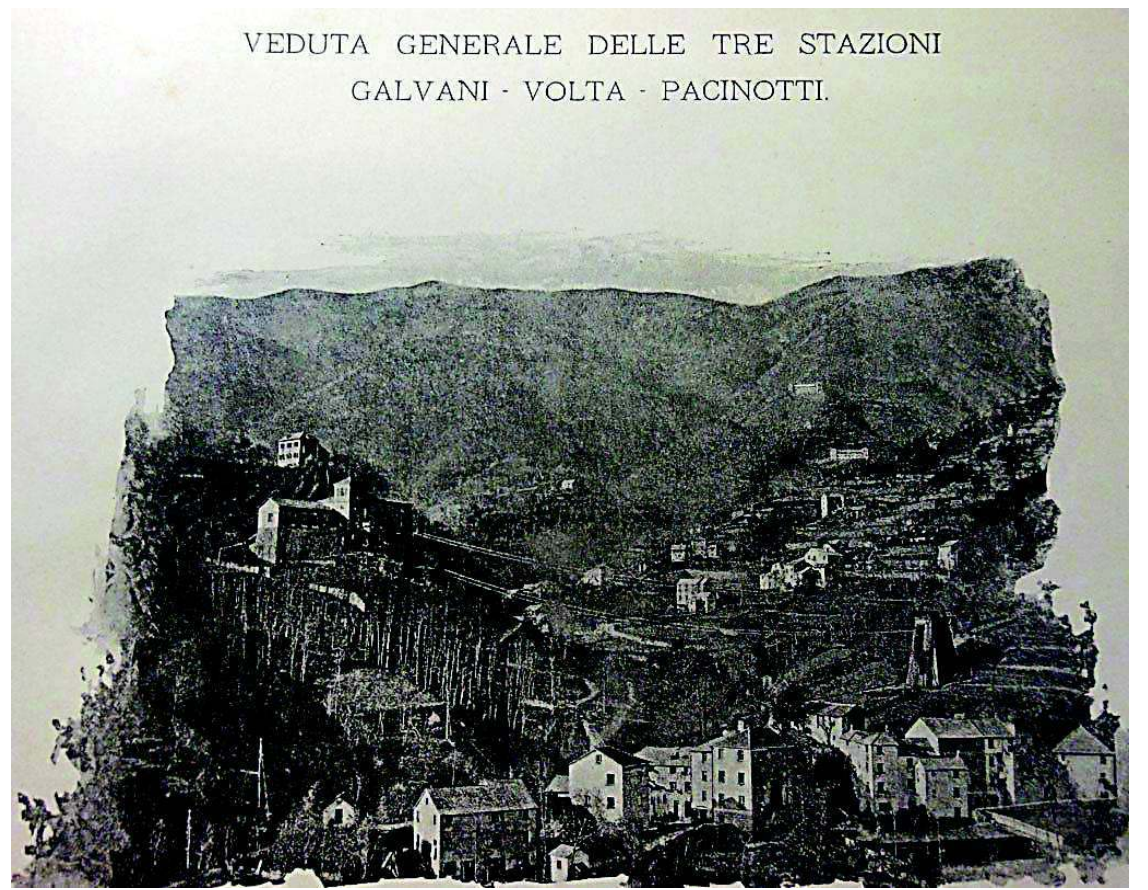

Figure 8: Planimétrie de la centrale Galvani en 1893 (BRUNO Niccolò, L'acquedotto De Ferrari Galliera, Hoepli, Milano, 1893. Vol II. Annexe XXVI).
La transmission de l'électricité, garantie par trois lignes aériennes distinctes (en fils métalliques et soutenues par des pieux en bois), apparaît comme la première exploitation de cette technique de distribution à grande échelle en Italie ${ }^{22}$ et en Europe. La première ligne de $16 \mathrm{~km}$ connecte la centrale Galvani au quartier de San Quirico, la seconde de $46 \mathrm{~km}$ la centrale Volta au centre-ville de Gênes, la dernière la centrale Pacinotti à la gare de Sampierdarena.

Les trois centrales garantissent en 1891 l'énergie à un premier groupe d'entreprises : Società Fratelli Montaldo (Pontedecimo) 25cv (chevaux-vapeur), Società Fratelli Cervetto (S. Quirico) 20cv, Società Fratelli Spallarossa (S. Quirico) $15 \mathrm{cv}$, Società Carlo Barabino \& C. (Bolzaneto) 20cv, Società fratelli Boccardo (Teglia) 35cv, signor Niccolò Castello (Teglia) 10cv, Società Italiana di Steaneria (Rivarolo) 20cv, Società Strade ferrate del mediterraneo (Rivarolo) 25cv, Società Strade Ferrate del Mediterraneo a (Sampierdarena) 40cv, Sig. Andrea Rebora (Sampierdarena) 40cv, Società Strade Ferrate del Mediterraneo a (Genova) 6ocv, pour une capacité totale de $410 \mathrm{cv}^{23}$. Parmi ces entreprises, on reconnaît des sociétés protagonistes de l'essor industriel de la vallée. Certaines d'elles ont ensuite acquis une notoriété nationale comme la Società Italiana di Steaneria, qui, dans ces années, sous la présidence de Erasmo Piaggio, occupe un site productif de $2300 \mathrm{~m}^{2}$ pour la production de savons et deviendra au siècle suivant, la Mira Lanza, une des principales entreprises italiennes dans le secteur des savons chimiques et des lessives ${ }^{24}$ ou la Strade Ferrate del Mediterraneo, une des deux sociétés créées en 1885 pour la gestion du réseau ferroviaire italien, nationalisée et intégrée en 1905 dans le Ferrovie dello Stato. 


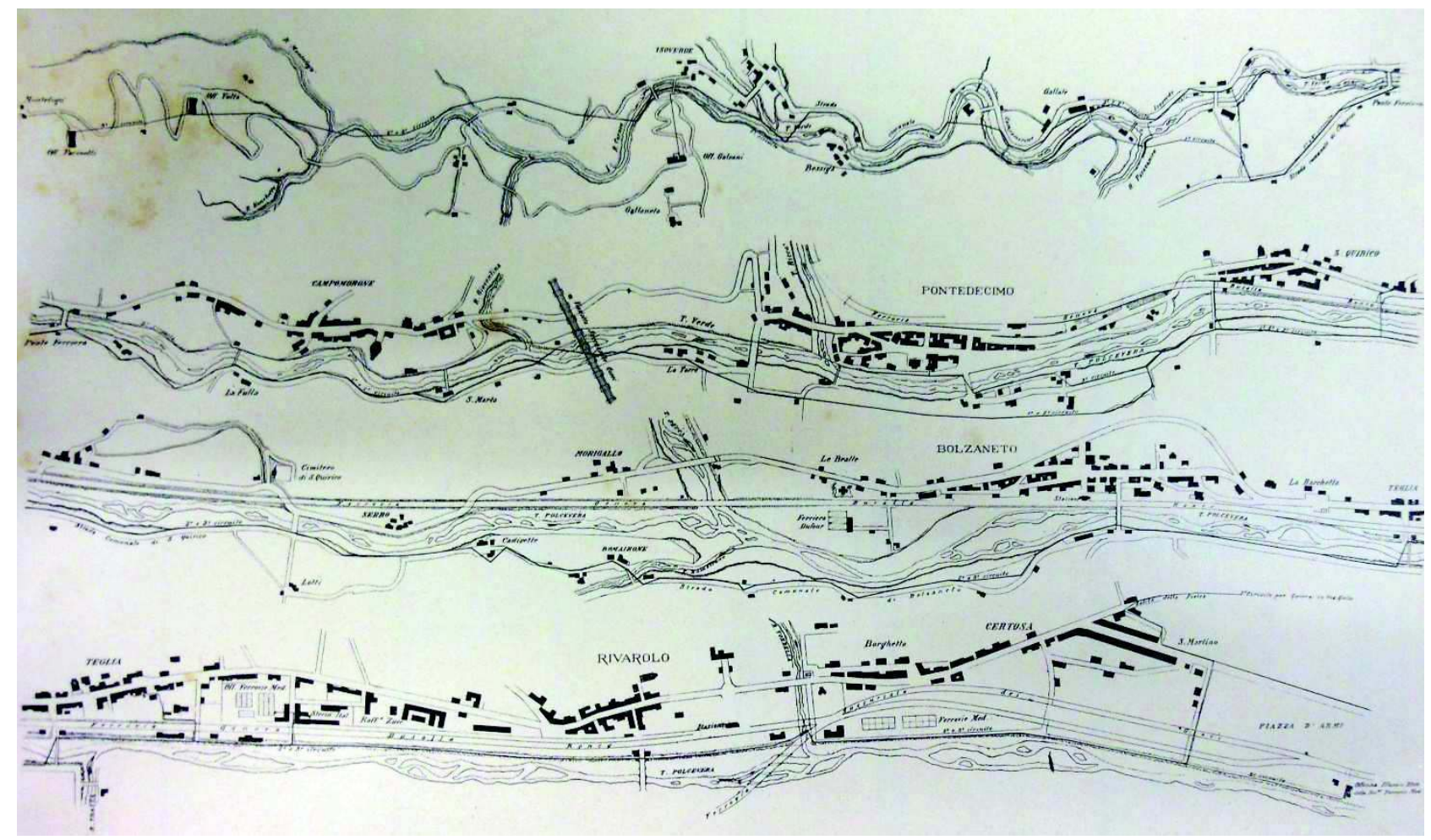

Figure 9: Schéma des circuits électriques.

Tiré de BRUNO Niccolò, L'acquedotto de Ferrari Galliera, Hoepli, Milano, 1893, vol. 2, annexe XXXIII.

$\mathrm{Au}$ début du $\mathrm{XX}^{\mathrm{e}}$ siècle, la Société De Ferrari Galliera décide la construction d'une nouvelle centrale hydroélectrique destinée à se substituer aux autres. Deux raisons essentielles à ces choix : les travaux sur les réservoirs qui avaient augmenté la portée en eau au-delà de la possibilité des centrales existantes ${ }^{25}$ et les nombreuses difficultés qui se manifestent dans la gestion du système de transmission ${ }^{26}$. Ceci est considéré par Giannetti comme un cas intéressant de "design impasse ", c'est-à-dire quand un paradigme technologique atteint une étape au-delà de laquelle chaque développement successif rencontre des difficultés et cause plus de problèmes que ceux qu'il résout 27.
Aujourd'hui, du système implanté à la fin du $\mathrm{XIX}^{\mathrm{e}}$ siècle, il ne subsiste que la centrale Galvani, située à proximité du village d'Isoverde, devenue une habitation privée, un des piliers utilisé pour la transmission de l'énergie télédynamique et la centrale Volta en ruines, dans les bois dans la montagne en surplomb des maisons d'Isoverde.

\section{La nouvelle centrale hydroélectrique}

Le plan élaboré par la société De Ferrari Galliera pour la rénovation du système de production d'énergie hydroélectrique destiné à tirer pleinement profit de la potentialité des bassins, résulte de 
l'évolution de l'ingénierie civile-qui, en 1905, semble avoir résolu le problème de la chute d'eau de 350 mètres :

« [...] les chutes d'eau de plus de 400 mètres, utilisé avec un saut unique en canalisation forcé, sont fort nombreuses. De celle de Chapareillan près de Chambéry de 612 mètres à celle de Gurtenellen près de Goschnem à l'entrée Nord du tunnel du Gottardo. [...] L'acier qui remplace la fonte a permis la même évolution dans les tuyaux que dans d'autres domaines de la construction métallique avec des tours de $300 \mathrm{~m}$ de hauteur et des ponts de $500 \mathrm{~m} » .{ }^{28}$

La nouvelle canalisation, réalisée avec des tuyaux en acier Martin Siemens, fournie par la société Ferrum de Kattowitz, est constituée d'une conduite forcée d'un diamètre de $750 \mathrm{~mm}$, sur une distance de 1340 mètres et une chute de $352 \mathrm{~m}$. La position idéale pour la nouvelle centrale est établie à proximité du torrent Chiappa dans une position isolée, à un kilomètre du village d'Isoverde; cet endroit, situé quasiment à la même altitude que la centrale Galvani, permet de réduire au maximum la distance entre la sortie du tunnel et la centrale, et ainsi les coûts des conduites forcées à installer. Les nouvelles turbines sont capables de générer une puissance quatre fois supérieure à celle produite par les trois centrales existantes ${ }^{29}$.

Le complexe de la nouvelle centrale se compose du bâtiment destiné à la production d'énergie hydroélectrique et d'un réservoir de compensation.

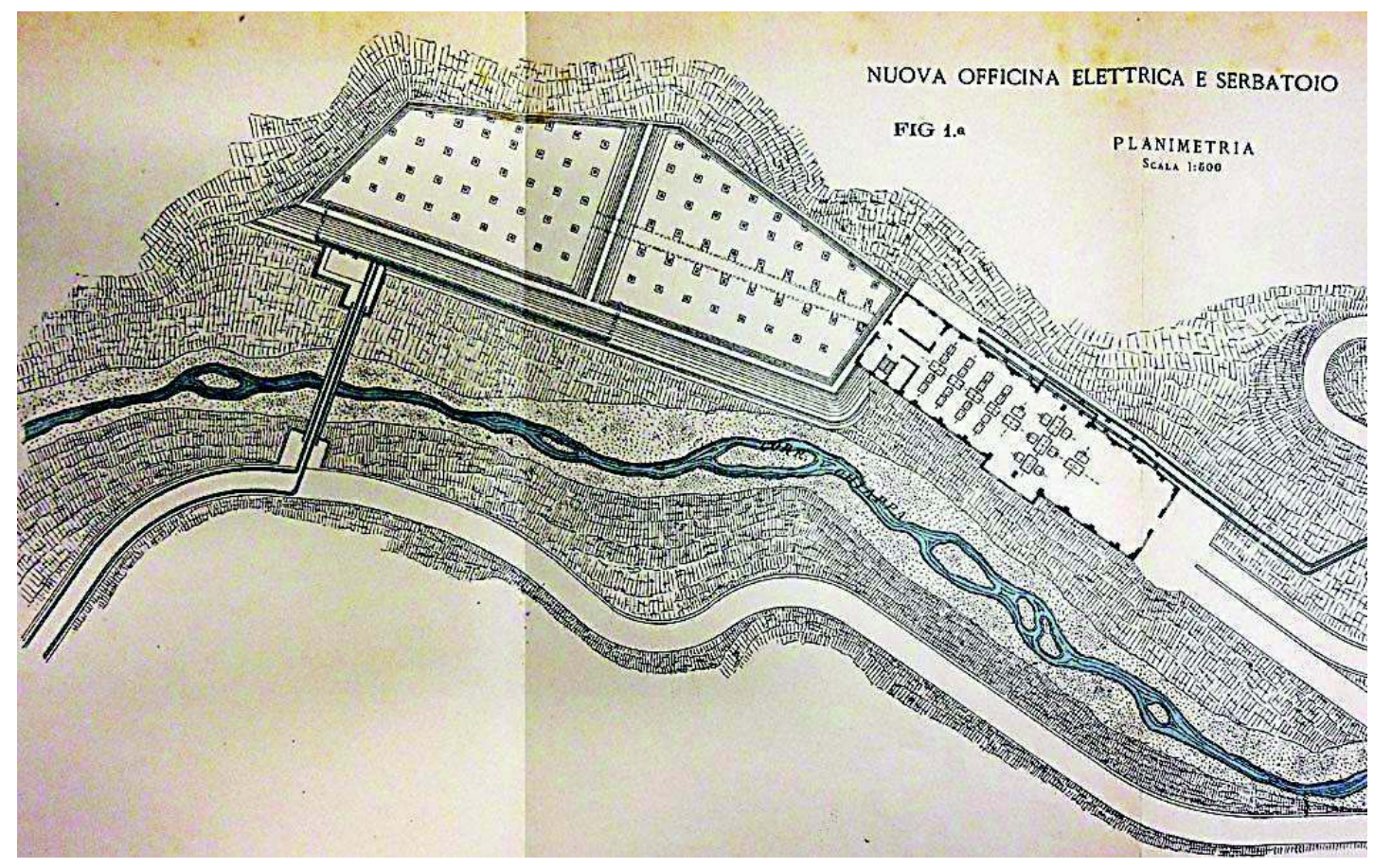

Figure 10 : Planimétrie de la nouvelle Centrale hydroélectrique d'Isoverde en 1904 (REGGIO Giacomo et BIGIO Antonio, Appendice alla monografia dell'ing. Niccolò Bruno sull'Acquedotto De Ferrari Galliera: Nuovi lavori eseguiti dalla società Acquedotto De Ferrari Galliera dopo l'anno 1893, Genova, 1904. Annexe II image 1) 


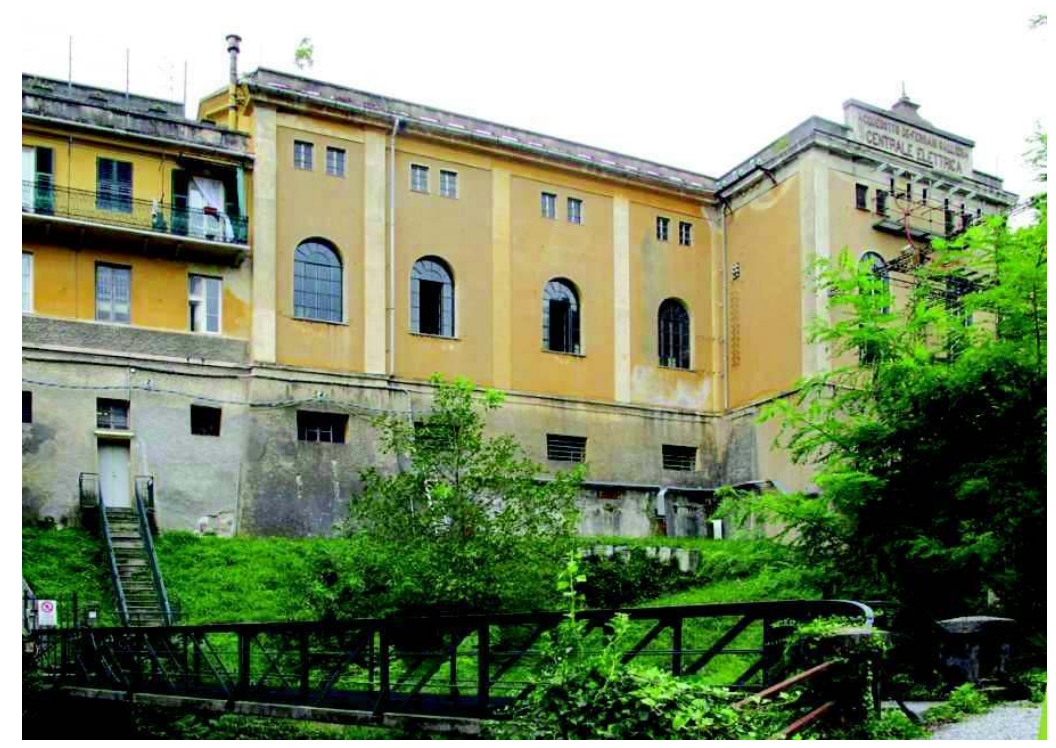

Figure 11: La nouvelle Centrale Hydroélectrique d'Isoverde en 2012 (photo Alberto Manzini, 2012).

Le bâtiment de la turbine mesure 49 x 15 mètres, et est réalisé en béton armé et en maçonnerie par la Società Anonima Cementi Armati di Genova, auquel s'ajoute un second élément de 14,5 x 9,5 mètres, destiné aux réparations ${ }^{30}$. Le complexe émerge avec force dans la verdure des bois; le volume central, surmonté d'une frise avec l'enseigne de la société, avance de la façade principale. Le sous-sol est traité comme un fondement sur lequel repose le rez-de-chaussée caractérisé par les pilastres en évidence et le double ordre de fenêtres (les fenêtres inférieures se terminent avec un arc en plein cintre, et à l'étage une séquence de petites ouvertures rectangulaires).

Proche de la centrale hydroélectrique est bâti un grand réservoir d'expansion divisé en deux parties. S'il avait au début du siècle la double fonction de réservoirs de compensation et de décanteurs pour la purification de l'eau; aujourd'hui, seule subsiste la fonction d'accumulation. La centrale d'Isoverde, complétée successivement avec un second réservoir, est aujourd'hui toujours en fonction.

Après la stagnation causée par la guerre, la Société De Ferrari Galliera participe à la constitution de la Société italienne Aqueduc Genovese, pour l'exploitation de l'aqueduc Nicolay. Dans les années Trente, les centrales hydroélectriques pour les barrages de Lavezze et Lavagnina sont mises en fonction, portant la capacité totale à $8000 \mathrm{~kW}$. L'expérience due à De Ferrari Galliera est suivie par d'autres barrages et lacs réalisés au cours des années suivantes, allant jusqu'à former un important réseau qui aujourd'hui encore garantit la fourniture d'eau de la ville et constitue un important producteur d'énergie électrique.

\section{De l'eau à l'énergie, des clés pour la compréhension d'un patrimoine et un territoire.}

Le patrimoine de l'eau, central pour ses implications sociales et productives, est désormais reconnu patrimoine commun et fait l'objet d'importants travaux de recherche. Aujourd'hui, 150 ans après le premier essai pour transporter l'eau du versant nord de l'Apennin à la ville, le système hydrique de Gênes, compte de nombreuses installations - bassins, canalisations, centres d'épuration de l'eau et centrale hydroélectrique et en particulier celle, sujet de notre étude qui ne représente qu'une infime partie et présente un intérêt historique et patrimonial constant qui va de la réutilisation à fins touristiques du parcours de l'aqueduc ancien et de la mise en valeur des plus importantes installations à la reconstitution de l'évolution des entreprises protagonistes de cette histoire. 
Notre contribution a voulu faire émerger des éléments patrimoniaux et historiques demeurés cachés du fait de leur brève existence compliquée et de leur localisation très isolée dans l'Apennin génois, afin de rétablir des connexions existantes autrefois, mais aujourd'hui moins perceptibles entre le ravitaillement d'eau de la ville, l'essor de l'industrie ligurienne, l'exigence d'énergie pour la production industrielle, la difficulté de distribution d'eau/d'énergie et la localisation des entreprises. En suivant ce chemin, du projet du De Ferrari Galliera jusqu'à la centrale hydroélectrique de 1905, nous percevons sous un angle diffèrent la disposition des bâtiments manufacturiers, les restes de vieilles centrales hydroélectriques et les tours de la ligne télédynamique. L'industrialisation - entendue comme un processus de transformation technoéconomique - et la modélisation du territoire effectuée par l'homme, deviennent alors non seulement compréhensibles mais donne à comprendre leur logique, et par ces clés de lecture nous permettent de transformer les éléments observés et restitués dans ces pages - des tours télédynamiques sans engin, des centrales hydroélectriques abandonnées dans la montagne, des centres de production entassés dans un territoire avare d'espace autrement dit des hiéroglyphes incompréhensibles - en un discours plus ample, qui nous présente ainsi des évènements qui ont touché cette vallée apenninique, et de manière plus générale la ville de Gênes, entre la fin du XIXe et le début du XX ${ }^{\mathrm{e}}$ siècle.

${ }^{1}$ DE MAESTRI Sara, TOLAINI Roberto, Storie e Itinerari dellindustria ligure, De Ferrari Editore, Genova, 2011, p. 191193 .

2 DORIA Marco, "Da un'economia di antico regime all'industrializzazione", dans ASSERETO Giovanni, DORIA Marco, dir., Storia della Liguria, Laterza, Roma-Bari, 2007, p. 211-212.
3 DORIA Marco, L'acqua e la città: Storia degli acquedotti genovesi De Ferrari Galliera e Nicolay (secoli XIX-XX), Franco Angeli, Milano, 2008.

4 PITTALUGA Michele, "Acqua che valica gli Appennini. Come nel 1853 un banchiere dissetò Genova con acqua padana”, dans Servizi a Rete, Marzo-Aprile, 2012, p. 81-82.

5 TEMPORELLI Giorgio,CASSINELLI Nicoletta, Gli acquedotti genovesi, Franco Angeli, Milano, 2007.

${ }^{6}$ DORIA Marco., op. cit., p. 49-58.

7 PITTALUGA Michele, op. cit., p. 83.

8 À propos de l'industrialisation de la Ligurie, nous renvoyons à : DORIA Marco, "Da un'economia di antico regime all'industrializzazione", dans ASSERETO Giovanni et DORIA Marco (dir.), Storia della Liguria, Laterza, Roma-Bari, 2007, p. 211-228.

9 Pour approfondir ce passage, dès les différentes propositions des années 1860, en particulier pour les éléments budgétaires et de l'ordre de l'ingénierie, à propos du choix du projet du Gorzente, nous renvoyons à : DORIA Marco, L'acqua e la città (...), p. 70-73.

10 Ils l'estiment à 1875 m³/h d'eau, par rapport à $1100 \mathrm{~m}^{3} / \mathrm{h}$ fourni par les aqueducs existants : $650 \mathrm{~m}^{3} / \mathrm{h}$ de l'aqueduc Civic (l'aqueduc historique) et $450 \mathrm{~m} 3 / \mathrm{h}$ de l'aqueduc de la Société Nicolay. Voir : TEMPORELLI Giorgio., op. cit, p. 60.

${ }^{11}$ BRUNO Niccolò, L'acquedotto De Ferrari Galliera, Hoepli, Milano, 1893, p. 20.

${ }^{12}$ Le territoire d'implantation des ouvrages de l'aqueduc, tant les barrages que les canalisations et les centrales, est localisé dans les communes de Campomorone et dans celles du Val Polcevera successivement englobées par la ville de Gênes.

${ }^{13}$ Les municipalités de la vallée d’Orba en Piémont, sur le côté nord des Apennins, qui jusque-là avaient utilisé les eaux du torrent Gorzente obtiennent la construction d'un réservoir de compensation à Lavagnina pour garantir la portée du torrent Gorzente aux villages de la vallée.

14 A.C.C. fald. 175, fasc 8.

${ }^{15}$ A.C.C., fald. 175 , fasc 8.

${ }^{16}$ DORIA Marco, L'acqua e la città (...), p. 76-78 ; p.89-92. 
${ }_{17}$ Raffaele De Ferrari, duc de Galliera (1803-1876), fut parmi les premiers dans le Royaume de Sardaigne à promouvoir la construction de chemins de fer et la mise en place de lignes maritimes. Entrepreneur et investisseur actif en France et en Italie, il est promoteur, en 1875 , des travaux pour l'amélioration du port avec un don de 20.000.00o de lires. L'utilité de cette œuvre touche ses contemporains qui lui ont aussi dédié la place centrale de la ville.

18 Par exemple, en 1888, pour le chantier de Lavezze les travailleurs sont 83 d'Alessandria (où passe le fleuve Gorzente), 50 de Belluno, 24 de Piacenza, 16 de Gênes, 14 de Padoue, 13 de Trévise, 7 de Pavia. Voir : ANGELINI Massimo, "Sviluppo industriale e società locale: Campomorone nell'ultimo quarto del XIX secolo", dans CAGNANA Aurora, dir., "Il paesaggio dell'uomo dai liguri 'Langanses' alla rivoluzione industriale", Studi e ricerche: cultura del territorio, numéro monographique, Comune di Campomorone, 1993.

${ }_{19}$ BRUNO Niccolò, op.cit., p. 123-127.

${ }^{20}$ BRUNO Niccolò, op.cit., p. 130.

${ }^{21}$ La méthode de distribution de l'énergie électrique en transmission du courant continu à haute tension dit «système série» est réalisée par le suisse René Thury en 1887. L'implantation de Gênes en représente la première expérimentation à grande échelle. À propos de René Thury, nous renvoyons à la fiche biographique réalisée par les archives de ville de Genève, disponible à l'adresse: http://w3public.villege.ch/seg/xmlarchives.nsfAttachments/thuryISAARframeset.ht $\mathrm{m} /$ file/thuryISAARframeset.htm?OpenElement, consulté en mars 2014.

${ }^{22}$ La centrale hydroélectrique d'Isoverde représente à l'époque une grande innovation: il s'agit de la première centrale hydroélectrique à courant continu réalisée en Italie. Voir FERRONE Vincenzo, dir., Torino Energia : Le politiche energetiche tra innovazione e società (1700-1930), Archivio Storico citta di Torino, 2007, p. 178.

23 BRUNO Niccolò, op. cit., p. 149.

24 DE MAESTRI Sara, TOLAINI Roberto, op. cit., p. 252-253.

${ }^{25}$ Après la première décennie de service, la Société De Ferrari Galliera poursuit son parcours d'amélioration des implantations : en 1891, le deuxième barrage est achevé à une altitude de 684 mètres, de $38,70 \mathrm{~m}$ de haut et de $220 \mathrm{~m}$ de long, avec la création du réservoir du lac, d'une capacité de 3,6 millions de $\mathrm{m}^{3}$.
Successivement sont réalisés le troisième bassin à une altitude de $717 \mathrm{~m}$ sur le torrent Badana (un affluent du Gorzente), par l'édification d'un barrage $(51,5 \mathrm{~m}$ de haut pour un développement de $216 \mathrm{~m}$ ) et la surélévation du barrage du lac Lavezze. Ces travaux permettent à l'ensemble des lacs du Gorzente d'atteindre une capacité totale de 11,5 millions de m³.

${ }^{26}$ Giannetti relève que le système adopté par la société De Ferrari Galliera, bien qu'ingénieux, présente de nombreuses limites, liées à l'irrégularité des utilisateurs, par exemple la différence d'usage entre le jour et la nuit ou entre les jours de la semaine, qui obligent à un interminable travail de réglage des installations. Voir à propos du fonctionnement des stations et des lignes de distribution : GIANNETTI Renato, « Tecnologia ed Economia del sistema elettrico », dans Storia dell'Industria elettrica in Italia, Vol. I, Laterza, Roma-Bari, 1992, p. 389-396.

27 GIANNETTI Renato, op. cit., p. 390. Pour la définition de design empasse adopté par Giannetti. Voir à propos de l'évolution du chemin de fer, DUFFY M. C., « Technomorphology and the Stephenson Traction System», Transaction of Newcomen Society, n. 54, 1982, p.55-59.

28 REGGIO Giacomo, BIGIO Antonio, Appendice alla monografia dell'ing. Niccolò Bruno sull'Acquedotto De Ferrari Galliera: Nuovi lavori eseguiti dalla società Acquedotto De Ferrari Galliera dopo l'anno 1893, Genova, 1904, p. 8.

29 TEMPORELLI Giorgio, op. cit., p. 93.

$3^{30}$ REGGIO Giacomo, BIGIO Antonio, op. cit., p.10. 


\section{Archives}

Archive de la mairie de Campomorone.

Archive de la fondation AMGA.

\section{Bibliographie}

AMGA, Acquedotto De Ferrari Galliera, 18801980, Sagep, Genova, 1980.

ASSERETO Giovanni, DORIA Marco, dir., Storia della Liguria, Laterza, Bari, 2007.

BRUNO Niccolò, L'acquedotto De Ferrari Galliera, Hoepli, Milano, 1893.

CAGNANA Aurora, dir., "Il paesaggio dell'uomo dai liguri 'Langanses' alla rivoluzione industriale", dans Studi e ricerche: cultura del territorio, Comune di Campomorone, 1993.

DE MAESTRI Sara, TOLAINI Roberto, Storie e Itinerari dell'industria ligure, De Ferrari Editore, Genova, 2011.

DORIA Giorgio, Investimenti e sviluppo economico a Genova alla vigilia della Prima Guerra Mondiale, vol I, 1815-1882, Giuffrè editoire, Milano, 1969.

DORIA Giorgio, Investimenti e sviluppo economico a Genova alla vigilia della Prima Guerra Mondiale, vol II, 1815-1882, Giuffrè editoire, Milano, 1969.

DORIA Marco, L'acqua e la città: Storia degli acquedotti genovesi De Ferrari galliera e Nicolay (secoli $X I X-X X$ ), Franco Angeli, Milano, 2008.

FERRONE Vincenzo, dir., Torino Energia: Le politiche energetiche tra innovazione e società (1700-1930), Archivio Storico citta di Torino, 2007.

GIANNETTI Renato, Tecnologia ed economia del sistema elettrico, in Storia dellindustria elettrica in Italia, vol I., Laterza, Roma-Bari, 1992.
GUASTONI Claudio, L'acquedotto Civico di Genova: un percorso al futuro, Franco Angeli, Milano, 2004.

PITTALUGA Michele, "Acqua che valica gli Appennini. Come nel 1853 un banchiere dissetò Genova con acqua padana", dans, Servizi a Rete, Marzo-Aprile, 2012.

REGGIO Giacomo, BIGIO Antonio, Appendice alla monografia delling. Niccolò Bruno sull'Acquedotto De Ferrari Galliera: Nuovi lavori eseguiti dalla società Acquedotto De Ferrari Galliera dopo l'anno 1893, Genova, 1904.

ROSSELLI Luciano, L'acquedotto storico di Genova: storia, architettura, mappe, fasi della costruzione: descrizione del percorso con itinerari storico-naturalistici, Genova, Nuova Editrice Genovese, 2009.

TEMPORELLI Giorgio, CASSINELLI Nicoletta, Gli acquedotti genovesi, Franco Angeli, Milano, 2007.

TEMPORELLI Giorgio, MANTELLI Francesco, L'acqua nella storia, Franco Angeli, Milano, 2007.

VINZONI Matteo, Piano dell'Acquedotto di Genova distinto nelle rispettive Custodie, Genova, Collection topographique de la mairie de Gênes, 1729.

VIOLLET Pierre-Luis, Histoire de l'énergie hydraulique : Moulins, pompes, roues et turbines de l'Antiquité au $X X^{e}$ siècle, Presse de l'École nationale des Ponts et Chaussées, Paris, 2005. 